\title{
An Automatable Formal Semantics for IEEE-754 Floating-Point Arithmetic
}

\author{
Martin Brain*, Cesare Tinelli $\dagger$, Philipp Rümmer ${ }^{\ddagger}$ and Thomas Wahl ${ }^{\S}$ \\ * Department of Computer Science, University of Oxford, Oxford, UK \\ $\dagger$ Department of Computer Science, The University of Iowa, Iowa City, IA, USA \\ $\ddagger$ Department of Information Technology, Uppsala University, Uppsala, Sweden \\ $\S$ College of Computer and Information Science, Northeastern University, Boston, MA, USA
}

\begin{abstract}
Automated reasoning tools often provide little or no support to reason accurately and efficiently about floating-point arithmetic. As a consequence, software verification systems that use these tools are unable to reason reliably about programs containing floating-point calculations or may give unsound results. These deficiencies are in stark contrast to the increasing awareness that the improper use of floating-point arithmetic in programs can lead to unintuitive and harmful defects in software. To promote coordinated efforts towards building efficient and accurate floating-point reasoning engines, this paper presents a formalization of the IEEE-754 standard for floating-point arithmetic as a theory in many-sorted first-order logic. Benefits include a standardized syntax and unambiguous semantics, allowing tool interoperability and sharing of benchmarks, and providing a basis for automated, formal analysis of programs that process floating-point data.
\end{abstract}

\section{INTRODUCTION}

Real values can be represented in a computer in many ways, with various level of precision: as fixed-point numbers, binary or decimal floating-point numbers, rationals, arbitrary precision reals, etc. Due to the wide availability of highperformance hardware and support in most programming languages, binary floating-point has become the dominant representation system. This creates a significant challenge for program analysis tools: accurate reasoning about the behavior of (numerical) programs is only possible with bit-accurate reasoning about floating-point arithmetic. Many automated verification tools, such as software model checkers, rely on solvers for Satisfiability Modulo Theories (SMT) [4] as their reasoning engines. These solvers use specialized, built-in methods to check the satisfiability of formulas in background theories of interest, such as for instance the theories of integer numbers, arrays, bit vectors and so on. Reasoning about floating-point numbers accurately in SMT then requires the identification of a suitable theory of floating-point arithmetic.

In the past, designing a formal theory of floating-point arithmetic would have been prohibitively complex, as different manufacturers used different floating-point formats which varied from the others in significant, structural aspects. The introduction, in 1985, and subsequent near universal adoption of the IEEE-754 standard has considerably improved the

Work partially supported by the Toyota Motor Corporation, ERC project 280053, EPSRC project EP/H017585/1, DSTL under CDE Project 30713, NSF grant CCF-1218075, and the VR grant 2011-6310. situation. However, the standard is unsatisfactory as a formal theory definition for a number of reasons: it is written in natural language; it covers various aspects that are not relevant to developing a theory of floating-point; it is lengthy (the 2008 revision has 70 pages) and, most critically for automated reasoning purposes, it does not describe a logical signature and interpretations.

This paper presents the syntax and semantics of a logical theory that formalizes floating-point arithmetic based on the IEEE-754 standard, with the goal of facilitating the construction of automated and precise reasoning tools for it. The models of our theory correspond exactly to conforming implementations of IEEE-754. While rather general, our formalization was developed with inputs from the SMT community to be a reference theory for SMT solver implementors and users, and was recently incorporated in the SMT-LIB standard [5], a widely used input/output language for SMT solvers.

This paper makes two specific contributions:

1) Presents mathematical structures intended to formally model binary floating-point arithmetic. [Section V]

2) Provides a signature for a theory of floating-point arithmetic and an interpretation of its operators in terms of the mathematical structures defined earlier. [Section VI]

\section{Floating-Point Arithmetic}

Floating-point refers to a way of encoding subsets of the rational numbers using bit vectors of fixed width. A floating point number consists of three such bit vectors: one for the fractional part of the number, called the significand, one for the exponent of an integer power by which the fractional part is multiplied, and a single bit for the sign. For example:

$$
\begin{aligned}
7.28125 & =7+28125 \cdot 10^{-5}=\left(2^{2}+2^{1}+2^{0}\right)+\left(2^{-2}+2^{-5}\right) \\
& =111.01001_{2}=1.1101001_{2} \cdot 2^{2}
\end{aligned}
$$

can be represented as a binary floating point number with a sign bit of 0 , an exponent of 2 and a significand of 1101001 : the leading 1 before the binary point is omitted (for so-called normal numbers) and hence known as the hidden bit.

Arithmetic on floating point numbers is defined as performing the operation as if the numbers were reals, followed by rounding the result to the nearest value representable as a floating point number; "nearest" is defined by a set of 
rules called a rounding mode. Many floating-point systems implemented in computer processors have included special values such as infinities and not-a-number $(\mathrm{NaN})$. When an underflow, overflow or other exceptional condition occurs, these special values can be returned instead of triggering an interrupt. This can simplify the control circuitry and results in faster computation but at the cost of making the floatingpoint number system more complex. IEEE-754 standardizes, in different floating-point formats, the sizes of the bit vectors used for number presentation, as well as the various rounding modes, and the meaning and use of special values.

\section{Formalization in Automated ReAsoning}

In general, to automate reasoning in a domain $\mathbf{D}$ of interest using a formal logic one has to restrict the set of possible interpretations assigned to the function and predicate symbols used to build formulas representing statements over $\mathbf{D}$. There are normally two approaches to achieve this, one axiomatic and one algebraic.

In the axiomatic approach, typical of interactive theorem proving tools, one first constructs a formula $\psi$ that axiomatizes D, i.e., formally describes (some of) the properties of the chosen function and predicate symbols. Then, to check that a particular formula $\phi$ is valid in $\mathbf{D}$ one asks the prover, in essence, to check the logical validity of the implication $\psi \Rightarrow \phi$. Previous formalizations of floating-point (see Section VII) have followed this approach. Since the axiomatization $\psi$ is part of the input, no specific support for the domain $\mathbf{D}$ on the prover's side is needed. Formalizations of this kind are flexible and easily extensible, however, the axioms $\psi$ tend to be voluminous and intricate, which can limit the performance of many automated techniques. Another limitation is that certain domains cannot be captured accurately by a relatively small, or even finite, axiomatization in the prover's logic. In that case, different, approximate axiomatizations may have to be considered and compared with respect the trade-offs they offer.

In the algebraic approach, typical of SMT solvers, a domain D is formalized instead by a set of algebraic structures (i.e., models in the chosen logic) that interpret the various functions and predicate symbols. The formalization is used as a specification for the prover, and the knowledge of what the symbols mean is pre-built into the prover. An advantage of this is that fast, domain specific procedures can be used to reason about $\mathbf{D}$. Moreover, in addition to checking for validity in $\mathbf{D}$, such procedure are usually also able to generate counterexamples for invalid formulas. Since these formalizations are used as specifications, the key issues are whether they can be implemented easily and efficiently and how well the interpretations they describe capture relevant properties of the domain.

We present a formalization of floating-point arithmetic in the algebraic style, intended as a specification for SMT solvers, and make the case that this accurately captures the semantics of the IEEE-754 standard. We concentrate on arithmetic aspects, abstracting away more operational ones, such as exception handling. Also, we only consider the case of binary
TABLE I

FORMALIZATION OF NaN's BEHAVIOR, WITH $u \in S^{*}$

$$
\begin{aligned}
& u+\mathrm{NaN}=\mathrm{NaN}+u=\mathrm{NaN} \quad-\mathrm{NaN}=\mathrm{NaN} \\
& u \cdot \mathrm{NaN}=\mathrm{NaN} \cdot u=\mathrm{NaN} \quad \mathrm{NaN}^{-1}=\mathrm{NaN} \\
& \mathrm{NaN} \leqslant u \Leftrightarrow u=\mathrm{NaN} \quad u \leqslant \mathrm{NaN} \Leftrightarrow u=\mathrm{NaN}
\end{aligned}
$$

(as opposed to decimal) floating-point arithmetic, as it is more widely used in practice.

\section{Formal Foundations}

IEEE-754 gives an informal definition of the semantics of floating-point operations:

Each of the computational operations that return a numeric result specified by this standard shall be performed as if it first produced an intermediate result correct to infinite precision and with unbounded range, and then rounded that intermediate result, if necessary, to fit in the destination's format.

To formalize this it is helpful to define an extension of the real numbers so that we can treat floating-point values as if they had "infinite precision and unbounded range," and to define a notion of rounding. Note that the extended reals are used here simply to aid the formal definition of floating-point operations. They are not the domain of interpretation of floating-point numbers. For that we will define a family of algebras over bit vector triples.

\section{A. Extended Reals}

We extend the set $\mathbb{R}$ of real numbers with three new elements: $+\infty,-\infty$ and $\mathrm{NaN}$, which represent respectively positive and negative infinity and a special not a number value used to obtain an arithmetically closed system. For each set $S \subseteq \mathbb{R}$ we can define the following sets:

$$
S^{\dagger}=S \cup\{+\infty,-\infty\} \quad S^{*}=S^{\dagger} \cup\{\mathrm{NaN}\}
$$

When $S$ is the base of an ordered additive or multiplicative monoid or group, we extend $\mathbb{R}$ 's binary operations + and , unary operations - and $\left({ }_{-}\right)^{-1}$ and order relation $\leq$. Table I gives axioms defining these operations when one argument is NaN. Table III gives axioms defining these operations when one argument is $+\infty$ or $-\infty$, as well as the axiom for the inverse of zero.

Note that $\left(\mathbb{R}^{*}, \leqslant\right)$ is a partial order since NaN is comparable only with itself. In contrast $\left(\mathbb{R}^{\dagger}, \leqslant\right)$ is a total order. The extended reals operate as three largely algebraically independent subsets: $\{\mathrm{NaN}\},\{+\infty,-\infty\}$ and $\mathbb{R}$. If a sub-expression of an expression $e$ evaluates to $\mathrm{NaN}$, then the whole $e$ evaluates to $\mathrm{NaN}$ - the set is closed under the basic operations. Infinities generate infinities or $\mathrm{NaN}$, although the reciprocal operator maps an infinity back to a real value. Reals are of course closed under all operations except reciprocal of zero. For convenience, we will also use the usual additional symbols in Table II, which are definable in terms of the basic operations. 
TABLE II

DEFINED SYMBOLS, WITH $x, y \in S^{*}$

$$
\begin{array}{rl}
x \geqslant y:=y \leqslant x \\
x-y:=x+(-y) & x<y:=(x \leqslant y) \wedge \neg(x=y) \\
x / y:=x \cdot y^{-1} & x>y:=(x \geqslant y) \wedge \neg(x=y)
\end{array}
$$

TABLE III

FORMALIZATION OF $\pm \infty$ 's AND INVERSE OF ZERO'S BEHAVIOR, $w \in S^{\dagger}$

$$
\begin{aligned}
& +\infty \leqslant w \Leftrightarrow w=+\infty \quad w \leqslant-\infty \Leftrightarrow w=-\infty \\
& w \leqslant+\infty \quad-(+\infty)=-\infty \quad+\infty^{-1}=0 \\
& -\infty \leqslant w \quad-(-\infty)=+\infty \quad-\infty^{-1}=0 \\
& w+(+\infty)=(+\infty)+w= \begin{cases}\mathrm{NaN} & \text { if } w=-\infty \\
+\infty & \text { if } w \neq-\infty\end{cases} \\
& w+(-\infty)=(-\infty)+w= \begin{cases}\mathrm{NaN} & \text { if } w=+\infty \\
-\infty & \text { if } w \neq-\infty\end{cases} \\
& w \cdot+\infty=+\infty \cdot w= \begin{cases}+\infty & \text { if } 0<w \\
-\infty & \text { if } w<0 \\
\mathrm{NaN} & \text { if } w=0\end{cases} \\
& w \cdot-\infty=-\infty \cdot w= \begin{cases}-\infty & \text { if } 0<w \\
+\infty & \text { if } w<0 \\
\mathrm{NaN} & \text { if } w=0\end{cases}
\end{aligned}
$$$$
0^{-1} \quad=+\infty
$$

\section{B. Rounding}

The second concept needed to formalize the IEEE-754 definition of operations is that of rounding; a map that will take the intermediate result in $\mathbb{R}^{*}$ back into the set of floatingpoint numbers. We define it as a function that selects between the two adjoints of the corresponding map into $\mathbb{R}^{*}$.

More generally, let $(X, \sqsubseteq)$ be a partially ordered set that consists of one or more disjoint lattices, and let $v: X \rightarrow \mathbb{R}^{*}$ be an order-embedding function from $X$ into the extended reals such that $\{+\infty,-\infty, \mathrm{NaN}\} \subset v(X)$. Then, the upper adjoint and lower adjoint of $v$ are respectively the unique functions $\bar{v}: \mathbb{R}^{*} \rightarrow X$ and $\underline{v}: \mathbb{R}^{*} \rightarrow X$ such that for all $r \in \mathbb{R}^{*}$.

- $r \leqslant v(\bar{v}(r))$ and $\bar{v}(r) \sqsubseteq x$ for all $x \in X$ with $r \leqslant v(x)$;

- $v(\underline{v}(r)) \leqslant r$ and $x \sqsubseteq \underline{v}(r)$ for all $x \in X$ with $v(x) \leqslant r$.

The function $\bar{v}$ maps an element $r$ to the smallest element of $X$ that projects above $r$ (rounding up) while $\underline{v}$ maps $r$ to the largest element of $X$ that projects below $r$ (rounding down). Let $\mathbb{B}=\{\top, \perp\}$ be the Booleans, with $\top$ being the true value. We define a family of (higher-order) rounding functions:

$$
\text { round }: \mathrm{RM} \times \mathbb{B} \times \mathbb{R}^{*} \rightarrow\left(X \rightarrow \mathbb{R}^{*}\right) \rightarrow\left(\mathbb{R}^{*} \rightarrow X\right)
$$

parametrized by the partially ordered set $X$, which provides a systematic way of selecting between rounding up and rounding down. Given a map $v: X \rightarrow \mathbb{R}^{*}$, the rounding function returns one of $v$ 's two adjoints, based on three previous inputs. The first is the rounding mode, chosen from the set:

$$
\mathrm{RM}=\{\text { rne, rna, rtp, rtn, rtz }\}
$$

which represents the five rounding modes defined by IEEE754 , namely, round to nearest with ties picking even value (rne), round to nearest with ties away from zero (rna), round
TABLE IV

DEFINITION OF round

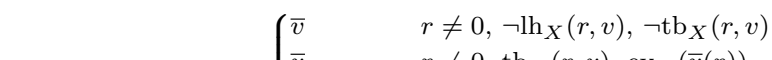

$$
\begin{aligned}
& \operatorname{round}(\operatorname{rne}, s, r)(v)= \begin{cases}\bar{v} & r \neq 0, \operatorname{tb}_{X}(r, v), \operatorname{ev}_{X}(\bar{v}(r)) \\
\underline{v} & r \neq 0, \operatorname{tb}_{X}(r, v), \operatorname{ev}_{X}(\underline{v}(r)) \\
\underline{v} & r \neq 0, \operatorname{lh}_{X}(r, v) \\
\operatorname{rsz}(s)(v) & r=0 \\
v & r=\mathrm{NaN}\end{cases}
\end{aligned}
$$

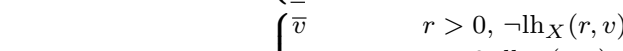

$$
\begin{aligned}
& \operatorname{round}(\mathrm{rna}, s, r)(v)= \begin{cases}\underline{v} & r>0, \mathrm{lh}_{X}(r, v) \\
\operatorname{rsz}(s)(v) & r=0 \\
\bar{v} & r<0, \neg \mathrm{lh}_{X}(r, v), \neg \operatorname{tb}_{X}(r, v) \\
\underline{v} & r<0, \mathrm{~h}_{X}(r, v) \vee \mathrm{b}_{X}(r, v)\end{cases} \\
& \underline{v} \quad r<0, \operatorname{lh}_{X}(r, v) \vee \operatorname{tb}_{X}(r, v) \\
& \operatorname{round}(\operatorname{rtp}, s, r)(v)= \begin{cases}\operatorname{rsz}(s)(v) & r=0 \\
\bar{v} & \text { otherwise }\end{cases} \\
& \operatorname{round}(\operatorname{rtn}, s, r)(v)= \begin{cases}\operatorname{rsz}(s)(v) & r=0 \\
\underline{v} & \text { otherwise }\end{cases} \\
& \operatorname{round}(\mathrm{rtz}, s, r)(v)= \begin{cases}\underline{v} & r>0 \\
\operatorname{rsz}(s)(v) & r=0 \\
\bar{v} & \text { otherwise }\end{cases} \\
& \text { where } \\
& \operatorname{rsz}(\top)(v)=\bar{v} \quad \operatorname{rsz}(\perp)(v)=\underline{v}
\end{aligned}
$$

towards $+\infty$ (rtp), round towards $-\infty$ (rtn), and round towards zero (rtz). The second input of round is a Boolean value determining the sign of zero when $X$ contains signed zeros (which is the case when $X$ is a set of floating-point numbers). The third input is the value to be rounded, needed because the rounding direction may depend on it (for example, when rounding to the nearest element of $X$ ).

The function round is defined in Table IV. The definition relies on three auxiliary predicates $\mathrm{lh}_{X}, \mathrm{tb}_{X}$ and $\mathrm{ev}_{X}$ whose own definition depend on the particular domain $X$. These express: when the value is in the lower half of the interval between two representations in $X$ (i.e. closer to $\underline{v}(r)$ than $\bar{v}(r)$ ); the tie-break condition when it is equal distance from both; and whether a representation is even. For illustration purposes, we provide a definition of those predicates in Table $\mathrm{V}$ for when $X=\mathbb{Z}$, the set of integers with the usual ordering. A definition of those predicates for sets of floating-point numbers is given later, after we formalize such sets.

The fairly elaborate definition of round is motivated by our goal to provide an accurate model of rounding as defined in IEEE-754. In particular, there is no mathematical reason for not using exclusively $\bar{v}$ or $\underline{v}$ in it. However, doing so would fail to reflect some properties of IEEE-754 floatingpoint numbers, for example "the sign of a sum [...] differs from at most one of the addends' signs" [1]. For brevity, we will write $\operatorname{rnd}(v, m, s, r)$ for the application $\operatorname{round}(m, s, r)(v)(r)$ which returns the value of $X$ that the real number $r$ is rounded to by using $v$.

\section{Models of Floating-Point ARithmetic}

In this section we specify a set of (many-sorted) structures in the sense of model theory. These are the intended models of 
TABLE V

AUXILIARY PREDICATES FOR ROUND IN THE CASE $X=\mathbb{Z}^{*}$

$$
\begin{aligned}
\operatorname{lh}_{\mathbb{Z}^{*}}(r, v) & :=r-v(\underline{v}(r))<v(\bar{v}(r))-r \\
\operatorname{tb}_{\mathbb{Z}^{*}}(r, v) & :=r-v(\underline{v}(r))=v(\bar{v}(r))-r \\
\operatorname{ev}_{\mathbb{Z}^{*}}(x) & :=\exists z \in \mathbb{Z} \cdot x=2 * z
\end{aligned}
$$

a logical theory of floating-point numbers that reflects IEEE754. In the next section we will specify a signature for such a theory and show how each sort, function and predicate symbol in the signature is interpreted over this set of structures.

\section{A. Universe}

The universe of each of our models consists of multiple sets: one for the rounding modes and one for each of the different floating-point precisions. Floating-point numbers other than $\mathrm{NaN}$ are triples of bit vectors modelling the three components (sign, exponent and significand) of the representations in IEEE-754. We identify bit vectors of length $\nu>0$ with elements of the function space $\mathbb{B} V_{\nu}=\mathbb{N}_{\nu} \rightarrow\{0,1\}$ where $\mathbb{N}_{\nu}=\{0, \ldots, \nu-1\}$. We write $\mathbf{1}_{\nu}$ for the unique function in $\mathbb{N}_{\nu} \rightarrow\{1\}$ and $\mathbf{0}_{\nu}$ for the unique function in $\mathbb{N}_{\nu} \rightarrow\{0\}$, which represent respectively the bit vector of length $\nu$ containing all ones and that containing all zeros. Let $\mathrm{ub}_{\nu}: \mathbb{B V}_{\nu} \rightarrow \mathbb{N}$ and $\mathrm{sb}_{\nu}: \mathbb{B} \mathbb{V}_{\nu} \rightarrow \mathbb{Z}$ denote the usual unsigned and 2's complement encodings of bit vectors into integers. Let $B_{\mu, \nu}$ denote the set $\mathbb{B V}_{1} \times \mathbb{B V}_{\mu} \times \mathbb{B V}_{\nu-1}$. For all integers $\varepsilon, \sigma>1$, we define the set of floating-point numbers with $\varepsilon$ exponent bits and $\sigma$ significand bits ${ }^{1}$ as the set $\mathbb{F}_{\varepsilon, \sigma}=\mathrm{F}_{\varepsilon, \sigma} \cup\{\mathrm{NaN}\}$ where

$$
\begin{aligned}
& \mathrm{F}_{\varepsilon, \sigma}=\mathrm{FZ}_{\varepsilon, \sigma} \cup \mathrm{FS}_{\varepsilon, \sigma} \cup \mathrm{FN}_{\varepsilon, \sigma} \cup \mathrm{FI}_{\varepsilon, \sigma} \\
& \mathrm{FZ}_{\varepsilon, \sigma}=\left\{(s, e, m) \in B_{\varepsilon, \sigma} \mid e=\mathbf{0}_{\varepsilon}, m=\mathbf{0}_{\sigma-1}\right\} \\
& \mathrm{FS}_{\varepsilon, \sigma}=\left\{(s, e, m) \in B_{\varepsilon, \sigma} \mid e=\mathbf{0}_{\varepsilon}, m \neq \mathbf{0}_{\sigma-1}\right\} \\
& \mathrm{FN}_{\varepsilon, \sigma}=\left\{(s, e, m) \in B_{\varepsilon, \sigma} \mid e \neq \mathbf{1}_{\varepsilon}, e \neq \mathbf{0}_{\varepsilon}\right\} \\
& \mathrm{FI}_{\varepsilon, \sigma}=\left\{(s, e, m) \in B_{\varepsilon, \sigma} \mid e=\mathbf{1}_{\varepsilon}, m=\mathbf{0}_{\sigma-1}\right\}
\end{aligned}
$$

The last four sets above correspond respectively to the bit vector triples used to represent zeros, subnormal numbers, normal numbers and infinities in IEEE-754, with the three components storing respectively sign, exponent and significand of the floating-point number. ${ }^{2}$ We will write informally -0 and +0 to refer to the two elements of $\mathrm{FZ}_{\varepsilon, \sigma}$.

We fix a total order $\sqsubseteq$ over $\mathrm{F}_{\varepsilon, \sigma}$ such that $\left(s_{1}, e_{1}, m_{1}\right) \sqsubseteq$ $\left(s_{2}, e_{2}, m_{2}\right)$ if one of the following holds:

- $s_{1}=1, s_{2}=0$

- $s_{1}=0, s_{2}=0, \mathrm{ub}_{\varepsilon}\left(e_{1}\right)<\mathrm{ub}_{\varepsilon}\left(e_{2}\right)$

- $s_{1}=0, s_{2}=0, \operatorname{ub}_{\varepsilon}\left(e_{1}\right)=\mathrm{ub}_{\varepsilon}\left(e_{2}\right), \mathrm{ub}_{\sigma}\left(m_{1}\right) \leqslant \mathrm{ub}_{\sigma}\left(m_{2}\right)$

- $s_{1}=1, s_{2}=1, \mathrm{ub}_{\varepsilon}\left(e_{2}\right)<\mathrm{ub}_{\varepsilon}\left(e_{1}\right)$

- $s_{1}=1, s_{2}=1, \operatorname{ub}_{\varepsilon}\left(e_{1}\right)=\operatorname{ub}_{\varepsilon}\left(e_{2}\right), \operatorname{ub}_{\sigma}\left(m_{2}\right) \leqslant \operatorname{ub}_{\sigma}\left(m_{1}\right)$

We extend $\sqsubseteq$ to a partial order on $\mathbb{F}_{\varepsilon, \sigma}$ by $\mathrm{NaN} \sqsubseteq \mathrm{NaN}$.

As discussed in Section IV, we define operations over $\mathbb{F}_{\varepsilon, \sigma}$ analogously to those defined over $\mathbb{R}^{*}$ by mapping floatingpoint values to extended reals, performing the corresponding

\footnotetext{
${ }^{1}$ Allowing arbitrary values for $\varepsilon$ and $\sigma$ is strictly a generalization of IEEE754 , which only defines a handful of precisions. However, doing so supports a wider range of applications with little additional notation and effort.

${ }^{2}$ The significand component has length $\sigma-1$ because the hidden bit, which is 1 for normal numbers, is not explicitly represented.
}

TABLE VI

AUXILIARY PREDICATES FOR ROUND IN THE CASE $X=\mathbb{F}_{\varepsilon, \sigma}$

$$
\begin{aligned}
\operatorname{ev}_{\mathbb{F}_{\varepsilon, \sigma}}(f):= & f=(s, e, m) \in \mathrm{F}_{\varepsilon, \sigma} \wedge \operatorname{ev}_{\mathbb{Z}^{*}}\left(\mathrm{ub}_{\sigma-1}(m)\right) \\
\operatorname{lh}_{\mathbb{F}_{\varepsilon, \sigma}}(r, \mathrm{v}):= & \sigma^{\prime}=\sigma+1 \wedge \mathrm{v}(\underline{\mathrm{v}}(r))=\mathrm{v}_{\varepsilon, \sigma^{\prime}}\left(\underline{\mathrm{v}_{\varepsilon, \sigma^{\prime}}}(r)\right) \\
\operatorname{tb}_{\mathbb{F}_{\varepsilon, \sigma}}(r, \mathrm{v}):= & \sigma^{\prime}=\sigma+1 \wedge \mathrm{v}(\underline{\mathrm{v}}(r))<\mathrm{v}_{\varepsilon, \sigma^{\prime}}\left(\underline{\mathrm{v}_{\varepsilon, \sigma^{\prime}}}(r)\right)= \\
& \mathrm{v}_{\varepsilon, \sigma^{\prime}}\left(\overline{\mathrm{v}_{\varepsilon, \sigma^{\prime}}}(r)\right)<\mathrm{v}_{\varepsilon, \sigma^{\prime}}(\overline{\mathrm{v}}(r))
\end{aligned}
$$

extended reals operation and then rounding the result back to a floating-point value. To formalize this we define a function $\mathrm{v}_{\varepsilon, \sigma}: \mathbb{F}_{\varepsilon, \sigma} \rightarrow \mathbb{R}^{*}$ which maps each floating-point number to the extended real it represents. Let $\operatorname{bias}(\varepsilon)=2^{\varepsilon-1}-1$.

$$
\begin{aligned}
& \mathrm{v}_{\varepsilon, \sigma}(f)=\mathrm{v}_{\varepsilon, \sigma}((s, e, m))= \\
& \begin{cases}0 & f \in \mathrm{FZ}_{\varepsilon, \sigma} \\
(-1)^{\mathrm{ub}_{1}(s)} \cdot 2^{1-\mathrm{bias}(\varepsilon)} \cdot\left(0+\frac{\mathrm{ub}_{\sigma-1}(m)}{2^{\sigma-1}}\right) & f \in \mathrm{FS}_{\varepsilon, \sigma} \\
(-1)^{\mathrm{ub}_{1}(s)} \cdot 2^{\mathrm{ub}_{\varepsilon}(e)-\operatorname{bias}(\varepsilon)} \cdot\left(1+\frac{\mathrm{ub}_{\sigma-1}(m)}{2^{\sigma-1}}\right) & f \in \mathrm{FN}_{\varepsilon, \sigma} \\
(-1)^{\mathrm{ub}_{1}(s)} \cdot(+\infty) & f \in \mathrm{FI}_{\varepsilon, \sigma}\end{cases} \\
& \mathrm{v}_{\varepsilon, \sigma}(\mathrm{NaN})=\mathrm{NaN}
\end{aligned}
$$

For brevity we will write just $\mathrm{v}$ in place of $\mathrm{v}_{\varepsilon, \sigma}$ when the values $\varepsilon$ and $\sigma$ are clear from context or not important. One can show that $\mathrm{v}$ is injective over $\mathbb{F}_{\varepsilon, \sigma} \backslash \mathrm{FZ}_{\varepsilon, \sigma}$ and monotonic. Thanks to the latter we have that both $\overline{\mathrm{v}}$ and $\mathrm{v}$ are well defined ${ }^{3}$ and so the function round can be used to map back from $\mathbb{R}^{*}$ to $\mathbb{F}_{\varepsilon, \sigma}$. The auxiliary predicates used in the definition of rounding in the case of $X=\mathbb{F}_{\varepsilon, \sigma}$ are defined in Table VI. Both $l_{\mathbb{F}_{\varepsilon, \sigma}}$ and $t b_{\mathbb{F}_{\varepsilon, \sigma}}$ use a set of floating-point numbers with one extra significand bit. This is equivalent to the guard bit used in hardware implementations, giving a point mid-way between $\overline{\mathrm{v}}$ and $\mathrm{v}$. The predicate $\operatorname{tb}_{\mathbb{F}_{\varepsilon, \sigma}}$ captures the property of $r$ being equidistant from $\mathrm{v}(r)$ and $\overline{\mathrm{v}}(r)$, which means that any further significand bits would be 0 . This is equivalent to the sticky bit used in hardware being equal to 0 .

\section{B. Relations}

Having defined a universe for the models, we next define various relations which will be used as the interpretation of predicates in the theory of floating-point. Every relation is parameterized by a floating-point domain, so each definition here actually describes a whole family of relations.

1) Unary Relations: We consider the following unary relations (subsets) for classifying floating-point numbers as well as determining their sign, if applicable. ${ }^{4}$

$$
\begin{aligned}
& \operatorname{isNeg}_{\varepsilon, \sigma}=\left\{f \in \mathrm{F}_{\varepsilon, \sigma} \mid f=(1, e, m)\right\} \\
& \operatorname{isPos}_{\varepsilon, \sigma}=\left\{f \in \mathrm{F}_{\varepsilon, \sigma} \mid f=(0, e, m)\right\}
\end{aligned}
$$

2) Binary Relations: We define a number of binary relations for comparing floating-point numbers. These are different from the equality and ordering relations on $\mathbb{F}_{\varepsilon, \sigma}$ (i.e., = and $\sqsubseteq$ ) and those on $\mathbb{R}^{*}$ (i.e., $=$ and $\leqslant$ ). Despite their names, they are not actually equality or ordering relations as they do

\footnotetext{
${ }^{3}$ These are surjections for all points except $\mathrm{FZ}_{\varepsilon, \sigma}$ which has the curious property that $-0=\overline{\mathrm{v}}(0) \sqsubseteq \underline{\mathrm{v}}(0)=+0$.

${ }^{4}$ These definitions imply $\bar{f}=\mathrm{NaN} \Leftrightarrow \neg\left(\operatorname{isNeg}_{\varepsilon, \sigma}(f) \vee \operatorname{isPos}_{\varepsilon, \sigma}(f)\right)$.
} 
not contain $(\mathrm{NaN}, \mathrm{NaN})$ and eq, leq and geq contain both $(+0,-0)$ and $(-0,+0)$.

$$
\begin{aligned}
\mathrm{eq}_{\varepsilon, \sigma} & =\left\{(f, g) \in \mathrm{F}_{\varepsilon, \sigma} \times \mathrm{F}_{\varepsilon, \sigma} \mid \mathrm{v}(f)=\mathrm{v}(g)\right\} \\
\operatorname{leq}_{\varepsilon, \sigma} & =\left\{(f, g) \in \mathrm{F}_{\varepsilon, \sigma} \times \mathrm{F}_{\varepsilon, \sigma} \mid \mathrm{v}(f) \leqslant \mathrm{v}(g)\right\} \\
\mathrm{lt}_{\varepsilon, \sigma} & =\left\{(f, g) \in \mathrm{F}_{\varepsilon, \sigma} \times \mathrm{F}_{\varepsilon, \sigma} \mid \mathrm{v}(f)<\mathrm{v}(g)\right\} \\
\operatorname{geq}_{\varepsilon, \sigma}=\left\{(f, g) \in \mathrm{F}_{\varepsilon, \sigma} \times \mathrm{F}_{\varepsilon, \sigma} \mid \mathrm{v}(f) \geqslant \mathrm{v}(g)\right\} & \mathrm{v}(f)>\mathrm{v}(g)\}
\end{aligned}
$$

\section{Operations}

Similarly to relations, we define families (parameterized by domains) of functions which will serve as the interpretation of various operations in the theory of floating-point.

a) Sign Operations: Two operations, negation and absolute value, manipulate the sign of the number. Since the domains of floating-point numbers are symmetric around 0 , there is no need for rounding and the operations can be defined directly on the floating-point bit vectors without using $\mathbb{R}^{*}$.

$$
\begin{aligned}
& \operatorname{neg}_{\varepsilon, \sigma}: \mathbb{F}_{\varepsilon, \sigma} \rightarrow \mathbb{F}_{\varepsilon, \sigma} \\
& \operatorname{neg}_{\varepsilon, \sigma}(f)= \begin{cases}(\neg s, e, m) & f=(s, e, m) \in \mathrm{F}_{\varepsilon, \sigma} \\
\mathrm{NaN} & f=\mathrm{NaN}\end{cases} \\
& \operatorname{abs}_{\varepsilon, \sigma}: \mathbb{F}_{\varepsilon, \sigma} \rightarrow \mathbb{F}_{\varepsilon, \sigma} \\
& \operatorname{abs}_{\varepsilon, \sigma}(f)= \begin{cases}(0, e, m) & f=(s, e, m) \in \mathrm{F}_{\varepsilon, \sigma} \\
\mathrm{NaN} & f=\mathrm{NaN}\end{cases}
\end{aligned}
$$

b) Arithmetic Operations: The main operations on floating-point numbers are those that correspond to the operations on an ordered field. They are defined by mapping arguments to $\mathbb{R}^{*}$ with $\mathrm{v}$, performing the corresponding operation in $\mathbb{R}^{*}$, and finally mapping the result back with round.

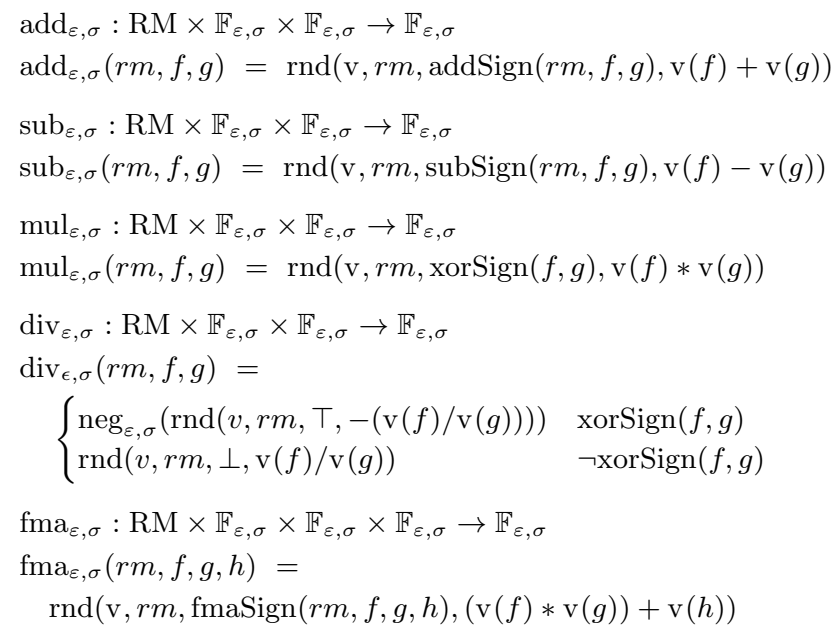

The addSign, subSign, xorSign and fmaSign predicates above are defined as follows ( $\oplus$ denotes exclusive or):

$$
\operatorname{addSign}(r m, f, g):= \begin{cases}\operatorname{isNeg}(f) \wedge \operatorname{isNeg}(g) & r m \neq \mathrm{rtn} \\ \operatorname{isNeg}(f) \vee \operatorname{isNeg}(g) & r m=\mathrm{rtn}\end{cases}
$$

$$
\operatorname{xorSign}(f, g):=\operatorname{isNeg}(f) \oplus \operatorname{isNeg}(g)
$$$$
\operatorname{fmaSign}(r m, f, g, h):=\operatorname{addSign}\left(r m, \operatorname{mul}_{\varepsilon, \sigma}(r m, f, g), h\right)
$$

Note that since $\mathrm{fma}_{\varepsilon, \sigma}$ only calls round once, it is not the same as $\operatorname{add}_{\varepsilon, \sigma}\left(r m, \operatorname{mul}_{\varepsilon, \sigma}(r m, a, b), c\right)$. Also, $\operatorname{div}_{\epsilon, \sigma}$ is equal to $\operatorname{rnd}(\mathrm{v}, r m, \operatorname{xorSign}(f, g), \mathrm{v}(f) / \mathrm{v}(g))$ at all points except positive numbers divided by -0 , where the "obvious" definition gives positive infinity while the definition given above gives the correct result of minus infinity.

c) Additional operations: Let $\mathrm{w}_{\varepsilon, \sigma}$ be the restriction of $\mathrm{v}_{\varepsilon, \sigma}$ to $\mathrm{FI}_{\varepsilon, \sigma} \cup\{\mathrm{NaN}\} \cup\left\{f \in \mathbb{F}_{\varepsilon, \sigma} \mid \mathrm{v}_{\varepsilon, \sigma}(f) \in \mathbb{Z}\right\}$. The following operation rounds back to a subset of $\mathbb{F}_{\varepsilon, \sigma}$, effectively mapping the value to the nearest whole number representable in the given floating-point format:

$$
\begin{aligned}
& \operatorname{rti}_{\varepsilon, \sigma}: \mathrm{RM} \times \mathbb{F}_{\varepsilon, \sigma} \rightarrow \mathbb{F}_{\varepsilon, \sigma} \\
& \operatorname{rti}_{\varepsilon, \sigma}(r m, f)=\operatorname{rnd}\left(\mathrm{w}_{\varepsilon, \sigma}, r m, \operatorname{isNeg}(f), \mathrm{v}(r)\right)
\end{aligned}
$$

Let in $: \mathbb{Z}^{*} \rightarrow \mathbb{R}^{*}$ with $\operatorname{in}(z)=z .^{5}$ Similarly to $\mathrm{rti}_{\varepsilon, \sigma}$, the remainder operation requires rounding an intermediate value to an integer:

$$
\begin{aligned}
& \operatorname{rem}_{\varepsilon, \sigma}: \mathrm{RM} \times \mathbb{F}_{\varepsilon, \sigma} \times \mathbb{F}_{\varepsilon, \sigma} \rightarrow \mathbb{F}_{\varepsilon, \sigma} \\
& \quad \begin{cases}f & g \in \mathrm{FI}_{\varepsilon, \sigma} \\
\mathrm{NaN} & f \in \mathrm{FI}_{\varepsilon, \sigma} \cup\{\mathrm{NaN}\} \\
\mathrm{NaN} & g \in \mathrm{FZ}_{\varepsilon, \sigma} \cup\{\mathrm{NaN}\} \\
\operatorname{rnd}(\mathrm{v}, r m, \operatorname{isNeg}(f), x) & x=\mathrm{v}(f)-(\mathrm{v}(g) * y), \\
& y=\operatorname{rnd}(\mathrm{in}, r m, 0, \mathrm{v}(f) / \mathrm{v}(g))\end{cases} \\
& \operatorname{remrne}_{\varepsilon, \sigma}: \mathbb{F}_{\varepsilon, \sigma} \times \mathbb{F}_{\varepsilon, \sigma} \rightarrow \mathbb{F}_{\varepsilon, \sigma} \\
& \operatorname{remrne}_{\varepsilon, \sigma}(f, g)=\operatorname{rem}_{\varepsilon, \sigma}(\mathrm{rne}, f, g)
\end{aligned}
$$

Note that the remainder computed as above is always exact when rne is used. This remainder function is the one used by the $\mathrm{C}$ standard library but is not necessarily the same as the intuitive idea of remainder which can be computed via: $\mathrm{fma}_{\varepsilon, \sigma}\left(r m, \operatorname{neg}_{\varepsilon, \sigma}\left(\operatorname{div}_{\varepsilon, \sigma}(r m, f, g)\right), g, f\right)$. The next two operations, the maximum and minimum of two floating-point numbers, are specified only partially: when the two arguments have the same value in $\mathbb{R}^{*}$, either one of the arguments can be returned.

$$
\begin{aligned}
& \max _{\varepsilon, \sigma}: \mathbb{F}_{\varepsilon, \sigma} \times \mathbb{F}_{\varepsilon, \sigma} \rightarrow \mathbb{F}_{\varepsilon, \sigma} \\
& \max _{\varepsilon, \sigma}(f, g)= \begin{cases}f & \mathrm{gt}_{\varepsilon, \sigma}(f, g) \text { or } g=\mathrm{NaN} \\
g & \mathrm{gt}_{\varepsilon, \sigma}(g, f) \text { or } f=\mathrm{NaN} \\
h & h \in\{f, g\}, \mathrm{eq}_{\varepsilon, \sigma}(f, g)\end{cases} \\
& \min _{\varepsilon, \sigma}: \mathbb{F}_{\varepsilon, \sigma} \times \mathbb{F}_{\varepsilon, \sigma} \rightarrow \mathbb{F}_{\varepsilon, \sigma} \\
& \min _{\varepsilon, \sigma}(f, g)= \begin{cases}f & \mathrm{lt}_{\varepsilon, \sigma}(f, g) \text { or } g=\mathrm{NaN} \\
g & \mathrm{lt}_{\varepsilon, \sigma}(g, f) \text { or } f=\mathrm{NaN} \\
h & h \in\{f, g\}, \mathrm{eq}_{\varepsilon, \sigma}(f, g)\end{cases}
\end{aligned}
$$

Note that the underspecification is an issue only when one of the inputs to $\max _{\varepsilon, \sigma}$ or $\min _{\varepsilon, \sigma}$ is -0 and the other is +0 .

${ }^{5}$ We consider $\mathbb{Z}$ a subset of $\mathbb{R}$ and hence $\mathbb{Z}^{*}$ a subset of $\mathbb{R}^{*}$. 
However, it means that we consider as acceptable models any structures with function families $\max _{\varepsilon, \sigma}$ and $\min _{\varepsilon, \sigma}$ that satisfy the specifications above, regardless of whether they return -0 or +0 for $(-0,+0)$, and for $(+0,-0)$. This is necessary because IEEE-754 itself allows either value to be returned, and compliant implementations do vary. For example, on some Intel processors the result returned by the $x 87$ and SSE units is different.

All the preceding operations have floating-point input and outputs in the same set, $\mathbb{F}_{\varepsilon, \sigma}$. To convert between different floating-point domains the following map is needed:

$$
\begin{aligned}
& \text { cast }_{\varepsilon, \sigma, \varepsilon^{\prime}, \sigma^{\prime}}: \mathrm{RM} \times \mathbb{F}_{\varepsilon^{\prime}, \sigma^{\prime}} \rightarrow \mathbb{F}_{\varepsilon, \sigma} \\
& \text { cast }_{\varepsilon^{\prime}, \sigma^{\prime}, \varepsilon, \sigma}(r m, f)=\operatorname{rnd}\left(\mathrm{v}_{\varepsilon, \sigma}, r m, \operatorname{isNeg}(f), \mathrm{v}_{\varepsilon^{\prime}, \sigma^{\prime}}(f)\right)
\end{aligned}
$$

If $\varepsilon \geqslant \varepsilon^{\prime}$ and $\sigma \geqslant \sigma^{\prime}$, the rounding mode argument is irrelevant since then all values of $\mathbb{F}_{\varepsilon^{\prime}, \sigma^{\prime}}$ are representable exactly in $\mathbb{F}_{\varepsilon, \sigma}$. However, this cannot be regarded as syntactic sugar because whether a value is a normal or a subnormal number does change depending on the floating-point domain.

\section{Combinations with Other Theories}

For many applications, the theory of floating-point is not sufficient to reason about the full problem; other theories such as integers, bit vectors, or reals are needed as well. This section describes the extensions to the intended models required to account for these additional domains, and possible mappings between them. IEEE-754 includes a number of functions to convert to "integer formats." We define here such conversions as well as extensions to IEEE-754 covering conversion to real and from real and bit vectors. Many of the additional operations are underspecified in that out of bounds and other error conditions do not have prescribed return values.

1) Real Numbers: For a model of the theory of floatingpoint to also be a model of the theory of reals, its universe has to be extended to a disjoint union with $\mathbb{R}$. Using the connections between $\mathbb{F}_{\varepsilon, \sigma}$ and $\mathbb{R}^{*}$, we add the following two conversion operations:

$$
\begin{aligned}
& \operatorname{realToFP}_{\varepsilon, \sigma}: \mathrm{RM} \times \mathbb{R} \rightarrow \mathbb{F}_{\varepsilon, \sigma} \\
& \operatorname{realToFP}_{\varepsilon, \sigma}(r m, r)=\operatorname{rnd}(\mathrm{v}, r m, 0, r) \\
& \text { FPToReal }_{\varepsilon, \sigma}: \mathbb{F}_{\varepsilon, \sigma} \rightarrow \mathbb{R} \\
& \text { FPToReal }_{\varepsilon, \sigma}(f)= \begin{cases}\mathrm{v}(f) & \mathrm{v}(f) \in \mathbb{R} \\
x & x \in \mathbb{R}, \text { otherwise }\end{cases}
\end{aligned}
$$

We do not specify what the value of $\operatorname{FPToReal}_{\varepsilon, \sigma}(f)$ is when $f$ does not correspond to a real number. This means again that we accept as a model any structure with a function family $\mathrm{FPToReal}_{\varepsilon, \sigma}$ that satisfies the specification above.

2) Fixed-size Bit Vectors: Similarly to the previous case, to form a joint model of the theories of floating-point and fixed-width bit vectors, the domain must be extended to a disjoint union with $\mathbb{B V}_{\nu}$ for every $\nu>0$. Let $\bullet: \mathbb{B} V_{\mu} \times$ $\mathbb{B} V_{\nu} \rightarrow \mathbb{B} V_{\mu+\nu}$ be the bit vector concatenation function for each $\mu, \nu>0$. The following function converts a bit vector of length $\varepsilon+\sigma$, with $\varepsilon, \sigma>1$, to a floating-point number in $\mathbb{F}_{\varepsilon, \sigma}$ by slicing the bit vector in three:

$$
\begin{aligned}
& \text { bitpatternToFP } \operatorname{Tof}_{\varepsilon, \sigma}: \mathbb{B V}_{\varepsilon+\sigma} \rightarrow \mathbb{F}_{\varepsilon, \sigma} \\
& \text { bitpatternToFP } \\
& \varepsilon, \sigma \\
& (b)= \begin{cases}(s, e, m) & b=s \bullet e \bullet m, \\
& (s, e, m) \in \mathrm{F}_{\varepsilon, \sigma} \\
\mathrm{NaN} & \text { otherwise }\end{cases}
\end{aligned}
$$

The function bitpatternToFP is not injective as there are multiple bit-patterns which represent NaN. This implies that it is not possible to give a reverse map without fixing the encoding of $\mathrm{NaN}$ to a particular value.

The next two functions first convert the bit vector to the integer value it denotes in binary, in 2's complement and unsigned format respectively, and then round that value to the corresponding floating-point. The last two functions do the inverse conversion.

$$
\begin{aligned}
& \operatorname{sIntToFP}_{\nu, \varepsilon, \sigma}: \mathrm{RM} \times \mathbb{B V}_{\nu} \rightarrow \mathbb{F}_{\varepsilon, \sigma} \\
& \operatorname{sIntToFP}_{\nu, \varepsilon, \sigma}(r m, b)=\operatorname{rnd}\left(\mathrm{v}, r m, 0, \operatorname{sb}_{\nu}(b)\right) \\
& \text { uIntToFP }{ }_{\nu, \varepsilon, \sigma}: \mathrm{RM} \times \mathbb{B V}_{\nu} \rightarrow \mathbb{F}_{\varepsilon, \sigma} \\
& \mathrm{uIntToFP}_{\nu, \varepsilon, \sigma}(r m, b)=\operatorname{rnd}\left(\mathrm{v}, r m, 0, \mathrm{ub}_{\nu}(b)\right) \\
& \text { FPToSInt }_{\nu, \varepsilon, \sigma}: \mathrm{RM} \times \mathbb{F}_{\varepsilon, \sigma} \rightarrow \mathbb{B V}_{\nu} \\
& \operatorname{FPToSInt}_{\nu, \varepsilon, \sigma}(r m, f)= \begin{cases}b & \mathrm{sb}_{\nu}(b)=\operatorname{rnd}(\mathrm{in}, r m, 0, \mathrm{v}(f)) \\
\mathrm{NaN} & \text { otherwise }\end{cases} \\
& \text { FPToUInt }_{\nu, \varepsilon, \sigma}: \mathrm{RM} \times \mathbb{F}_{\varepsilon, \sigma} \rightarrow \mathbb{B V}_{\nu} \\
& \text { FPToUInt }_{\nu, \varepsilon, \sigma}(r m, f) \begin{cases}b & \mathrm{ub}_{\nu}(b)=\operatorname{rnd}(\mathrm{in}, r m, 0, \mathrm{v}(f)) \\
\mathrm{NaN} & \text { otherwise }\end{cases}
\end{aligned}
$$

\section{From MODELS TO THEORY}

We now formalize a logical theory of floating-point numbers based on the structures defined in the previous section. We use the version of many-sorted logic adopted by the SMT-LIB 2 standard [5].

A (logical) signature is consists of a set of sort symbols (of arity $\geq 0)$ and a set of function symbols $f$ with an associated rank, a tuple $\left(S_{1}, \ldots, S_{n}, S\right)$ of sort terms specifying the sort of $f$ 's arguments, namely, $S_{1}, \ldots, S_{n}$, and result, $S$. Constants are represented by nullary function symbols; predicate symbols by function symbols whose return sort is a distinguished sort Bool. Every signature $\Sigma$ is assumed to contain Bool and constants true and false of that sort, as well as an overloaded symbol $=$ of $\operatorname{rank}(S, S$, Bool $)$ for each sort $S$, for the identity relation over $S$. Given a set of sorted variables for each of the sorts in $\Sigma$, well-sorted terms and well-sorted formulas of signature $\Sigma$ are defined as usual.

For every signature $\Sigma$, a $\Sigma$-interpretation $\mathcal{I}$ interprets each sort $S$ in $\Sigma$ as a non-empty set $\llbracket S \rrbracket_{\mathcal{I}}$, each variable $x$ of sort $S$ as an element $\llbracket x \rrbracket_{\mathcal{I}}$ of $\llbracket S \rrbracket_{\mathcal{I}}$, and each function symbol $f$ of rank $\left(S_{1}, \ldots, S_{n}, S\right)$ as an element $\llbracket f \rrbracket_{\mathcal{I}}$ of the (total) function space $\llbracket S_{1} \rrbracket_{\mathcal{I}} \times \cdots \times \llbracket S_{n} \rrbracket_{\mathcal{I}} \rightarrow \llbracket S \rrbracket_{\mathcal{I}}$. Additionally, $\mathcal{I}$ interprets Bool as $\mathbb{B}=\{\top, \perp\}$ and each $=$ of $\operatorname{rank}(S, S$, Bool $)$ as the function that maps $(x, y) \in S \times S$ to $\top$ iff $x$ is $y$. For 
TABLE VII

SORTS AND THEIR INTERPRETATION REQUIREMENTS

$\begin{aligned} \llbracket \text { RoundingMode }_{\mathcal{I}} & =\mathrm{RM} & \llbracket \text { FloatingPoint }_{\varepsilon, \sigma} \rrbracket_{\mathcal{I}} & =\mathbb{F}_{\varepsilon, \sigma} \\ \llbracket \text { Real } \rrbracket_{\mathcal{I}} & =\mathbb{R} & \llbracket \text { BitVec }_{\nu} \rrbracket_{\mathcal{I}} & =\mathbb{B} V_{\nu}\end{aligned}$

TABLE VIII

CONSTRUCTOR SYMBOLS AND THEIR INTERPRETATION

Symbols of rank RM:

$$
\begin{aligned}
& \llbracket r n e \rrbracket_{\mathcal{I}}=\text { rne } \quad \llbracket r n a \rrbracket_{\mathcal{I}}=\text { rna } \quad \llbracket r t p \rrbracket_{\mathcal{I}}=\text { rtp } \\
& \llbracket r \operatorname{tn} \rrbracket_{\mathcal{I}}=\mathrm{rtn} \quad \llbracket r \mathrm{rtz} \rrbracket_{\mathcal{I}}=\mathrm{rtz}
\end{aligned}
$$

Symbols of rank $\mathrm{FP}_{\varepsilon, \sigma}$ :

$$
\begin{aligned}
& \llbracket+\infty 0_{\varepsilon, \sigma} \rrbracket_{\mathcal{I}}=\left(0, \mathbf{1}_{\varepsilon}, \mathbf{0}_{\sigma-1}\right) \quad \llbracket+\text { zero }_{\varepsilon, \sigma} \rrbracket_{\mathcal{I}}=\left(0, \mathbf{0}_{\varepsilon}, \mathbf{0}_{\sigma-1}\right) \\
& \llbracket-\circ 0_{\varepsilon, \sigma} \rrbracket_{\mathcal{I}}=\left(1, \mathbf{1}_{\varepsilon}, \mathbf{0}_{\sigma-1}\right) \quad \llbracket \text {-zero } \varepsilon_{\varepsilon, \sigma} \rrbracket_{\mathcal{I}}=\left(1, \mathbf{0}_{\varepsilon}, \mathbf{0}_{\sigma-1}\right) \\
& \llbracket \mathrm{NaN}_{\varepsilon, \sigma} \rrbracket_{\mathcal{I}}=\mathrm{NaN} \\
& \text { Symbols of rank }\left(\mathrm{BV}_{1}, \mathrm{BV}_{\varepsilon}, \mathrm{BV}_{\sigma}, \mathrm{FP}_{\varepsilon, \sigma}\right) \text { : } \\
& \llbracket \mathrm{fp} \rrbracket_{\mathcal{I}}=\lambda\left(b_{1}, b_{\varepsilon}, b_{\sigma-1}\right) . \text { bitpatternToFP } \operatorname{P}_{\varepsilon, \sigma}\left(b_{1} \bullet b_{\varepsilon} \bullet b_{\sigma-1}\right)
\end{aligned}
$$

each sort $S, \mathcal{I}$ induces a mapping $\llbracket_{-} \rrbracket_{\mathcal{I}}$ from terms of sort $S$ to $\llbracket S \rrbracket_{\mathcal{I}}$ as expected. A satisfaction relation $\models$ between $\Sigma$ interpretations and $\Sigma$-formulas is also defined as expected. A theory of signature $\Sigma$ as a pair $T=(\Sigma, \mathbf{I})$ where $\mathbf{I}$ is a set of $\Sigma$-interpretations, the models of $T$, that is closed under variable reassignment. We say that a $\Sigma$-formula $\varphi$ is satisfiable (resp., unsatisfiable) in $T$ if $\mathcal{I} \models \varphi$ for some (resp., no) $\mathcal{I} \in \mathbf{I}$.

\section{A. A Theory of Floating-Point Numbers}

In the following we define a theory $T_{\mathrm{FP}}$ of floating-point numbers in the sense above by specifying a signature $\Sigma_{\mathrm{FP}}$ and a set of $\mathbf{I}_{\mathrm{FP}}$ of $\Sigma_{\mathrm{FP}}$-interpretations.

The sorts of $\Sigma_{\mathrm{FP}}$ consist, besides Bool, of two individual sorts: RoundingMode and Real; and two sort families: $\mathrm{BitVec}_{\nu}$, indexed by an integer $\nu>0$, and FloatingPoint $_{\varepsilon, \sigma}$, indexed by two integers $\varepsilon, \sigma>1$. The set of function symbols of $\Sigma_{\mathrm{FP}}$, and their ranks, is given in Table VIII through $\mathrm{X}$. In those tables, we abbreviate RoundingMode, FloatingPoint, and BitVec respectively as $\mathrm{RM}, \mathrm{FP}$, and $\mathrm{BV}$.

We define the set $\mathbf{I}_{\mathrm{FP}}$ as the set of all possible $\Sigma_{\mathrm{FP}}$ interpretations $\mathcal{I}$ that interpret sort and function symbol as specified in Table VII through $\mathrm{X}$ in terms of the sets and functions introduced in Section V. Many of the function symbols are overloaded for having different ranks; so we specify their interpretation separately for each rank.

As shown in Table VIII, the theory has a family of symbols denoting the floating-point infinities, zeros, and $\mathrm{NaN}$ for each pair of exponent and significand length. It also has a ternary function symbol $\mathrm{fp}$ that constructs a floating point number from a triple of bit vectors respectively storing the sign, exponent and significant. This allows us to represent all nonNaN values with bit-level precision.

Table IX lists function symbols for the various arithmetic operations over floating-point numbers, and provides their se-
TABLE IX

MAIN SYMBOLS AND THEIR INTERPRETATION

$$
\begin{aligned}
& \text { Symbols of rank }\left(\mathrm{FP}_{\varepsilon, \sigma}, \mathrm{FP}_{\varepsilon, \sigma}\right) \text { : } \\
& \llbracket f \mathrm{f} . \mathrm{abs} \rrbracket_{\mathcal{I}}=\operatorname{abs}_{\varepsilon, \sigma} \quad \llbracket f \mathrm{f} . n \operatorname{neg} \rrbracket_{\mathcal{I}}=\operatorname{neg}_{\varepsilon, \sigma} \\
& \text { Symbols of rank }\left(\mathrm{FP}_{\varepsilon, \sigma}, \mathrm{FP}_{\varepsilon, \sigma}, \mathrm{FP}_{\varepsilon, \sigma}\right) \text { : } \\
& \llbracket \mathrm{fp} \cdot \max \rrbracket_{\mathcal{I}}=\max _{\varepsilon, \sigma} \quad \text { ffp.min } \rrbracket_{\mathcal{I}}=\min _{\varepsilon, \sigma} \\
& \text { Symbols of rank }\left(\mathrm{RM}, \mathrm{FP}_{\varepsilon, \sigma}, \mathrm{FP}_{\varepsilon, \sigma}\right) \text { : } \\
& \llbracket \text { fp.roundToIntegral } \rrbracket_{\mathcal{I}}=\mathrm{rti}_{\varepsilon, \sigma} \\
& \llbracket f \text { p. } \operatorname{add} \rrbracket_{\mathcal{I}}=\operatorname{add}_{\varepsilon, \sigma} \quad \llbracket \text { fp.sub } \rrbracket_{\mathcal{I}}=\operatorname{sub}_{\varepsilon, \sigma} \\
& \llbracket f \text { p.mul } \rrbracket_{\mathcal{I}}=\operatorname{mul}_{\varepsilon, \sigma} \quad \llbracket f \text { p.div } \rrbracket_{\mathcal{I}}=\operatorname{div}_{\varepsilon, \sigma}
\end{aligned}
$$

$$
\llbracket \mathrm{fp} . \mathrm{fma} \rrbracket_{\mathcal{I}}=\mathrm{fma}_{\varepsilon, \sigma}
$$

Symbols of rank $\left(\mathrm{FP}_{\varepsilon, \sigma}, \mathrm{Bool}\right)$ :

$$
\begin{aligned}
& \llbracket \text { fp.isNormal } \rrbracket_{\mathcal{I}}=\mathrm{FN}_{\varepsilon, \sigma} \quad \text { [fp.isNegative } \rrbracket_{\mathcal{I}}=\operatorname{isNeg}_{\varepsilon, \sigma} \\
& \llbracket \text { fp.isSubnormal } \rrbracket_{\mathcal{I}}=\mathrm{FS}_{\varepsilon, \sigma} \quad \llbracket \text { fp.isPositive } \rrbracket_{\mathcal{I}}=\operatorname{isPos}_{\varepsilon, \sigma} \\
& \llbracket \text { fp.isInfinite } \rrbracket_{\mathcal{I}}=\mathrm{FI}_{\varepsilon, \sigma} \quad \text { [fp.isZero } \rrbracket_{\mathcal{I}}=\mathrm{FZ}_{\varepsilon, \sigma} \\
& \llbracket \mathrm{fp} . \mathrm{isNaN} \rrbracket_{\mathcal{I}}=\{\mathrm{NaN}\} \\
& \llbracket \mathrm{fp} .1 \mathrm{t} \rrbracket_{\mathcal{I}}=\mathrm{lt}_{\varepsilon, \sigma} \quad \llbracket \mathrm{fp} . \mathrm{leq}_{\mathcal{I}}=\mathrm{leq}_{\varepsilon, \sigma} \\
& \llbracket \mathrm{fp} . \mathrm{gt} \rrbracket_{\mathcal{I}}=\mathrm{gt}_{\varepsilon, \sigma} \quad \llbracket \mathrm{fp} . \mathrm{geq} \rrbracket_{\mathcal{I}}=\mathrm{geq}_{\varepsilon, \sigma} \\
& \llbracket \text { fp.eq } \rrbracket_{\mathcal{I}}=\mathrm{eq}_{\varepsilon, \sigma}
\end{aligned}
$$

TABLE $\mathrm{X}$

CONVERSION SYMBOLS AND THEIR INTERPRETATION

Conversions to floating-point

$$
\begin{aligned}
& \llbracket \text { to_f } \mathrm{p}_{\varepsilon, \sigma}:\left(\mathrm{RM}, \mathrm{FP}_{\mathcal{\varepsilon}^{\prime}, \sigma^{\prime}}, \mathrm{FP}_{\varepsilon, \sigma}\right) \rrbracket_{\mathcal{I}}=\text { cast }_{\mathcal{\varepsilon}^{\prime}, \sigma^{\prime}, \varepsilon, \sigma} \\
& \llbracket \text { to_f } \mathrm{p}_{\varepsilon, \sigma}:\left(\mathrm{BV}_{\varepsilon+\sigma}, \mathrm{FP}_{\varepsilon, \sigma}\right) \rrbracket_{\mathcal{I}}=\text { bitpatternToFP }{ }_{\varepsilon, \sigma} \\
& \llbracket \text { to_f } \mathrm{f}_{\varepsilon, \sigma}:\left(\mathrm{RM}, \operatorname{Real}, \mathrm{FP}_{\varepsilon, \sigma}\right) \rrbracket_{\mathcal{I}}=\operatorname{realToFP}_{\varepsilon, \sigma} \\
& \text { 【to_f } \mathrm{p}_{\varepsilon, \sigma}:\left(\mathrm{RM}, \mathrm{BV}_{\nu}, \mathrm{FP}_{\varepsilon, \sigma}\right) \rrbracket_{\mathcal{I}}=\operatorname{sintToFP}_{\nu, \varepsilon, \sigma} \\
& \text { 【to_fp_unsigned } d_{\varepsilon, \sigma}:\left(\mathrm{RM}_{1} \mathrm{BV}_{\nu}, \mathrm{FP}_{\varepsilon, \sigma}\right) \rrbracket_{\mathcal{I}}=\mathrm{uIntToFP}_{\nu, \varepsilon, \sigma}
\end{aligned}
$$

Conversions from floating-point

$$
\begin{aligned}
\llbracket \text { fp.to_sbv } & :\left(\mathrm{FP}_{\varepsilon, \sigma}, \mathrm{BV}_{\nu}\right) \rrbracket_{\mathcal{I}}=\mathrm{FPToSInt}_{\nu, \varepsilon, \sigma} \\
\llbracket \text { fp.to_ubv }_{\nu}:\left(\mathrm{FP}_{\varepsilon, \sigma}, \mathrm{BV}_{\nu}\right) \rrbracket_{\mathcal{I}} & =\mathrm{FPToUInt}_{\nu, \varepsilon, \sigma} \\
\llbracket \text { fp.to_real }:\left(\mathrm{FP}_{\varepsilon, \sigma}, \mathrm{Real}\right) \rrbracket_{\mathcal{I}} & =\mathrm{FPToReal}_{\nu, \varepsilon, \sigma}
\end{aligned}
$$

mantics in terms of the operations defined in Subsection V-C. The table lists predicate symbols corresponding to the relations defined in Subsection V-B and to the various subsets of $\mathbb{F}_{\varepsilon, \sigma}$. For simplicity and by a slight abuse of notation, we identify functions $h$ of type $D_{1} \times \cdots \times D_{n} \rightarrow \mathbb{B}$ with the $n$-ary relations $\left\{\left(x_{1}, \ldots, x_{n}\right) \in D_{1} \times \cdots \times D_{n} \mid h\left(x_{1}, \ldots, x_{n}\right)=\top\right\}$.

Finally, Table $\mathrm{X}$ lists function symbols corresponding to the various conversion functions introduced in Subsection V-D as well as the casting function between floating-point sets of different precision. 


\section{RELATED WORK}

The earliest formalizations of floating-point [10] were limited by the diversity of floating-point formats and systems they had to cover. For example, the formalization needed to support a range of bases, as 2, 16 and 10 were all in use. Overflow and underflow were particularly problematic as, again, systems in common usage took very different approaches to handling them. The first formalization of IEEE-754 [6] is notable on several grounds. It was the first to use a formal language $(\mathrm{Z})$ and to be used to verify algorithms for the basic operations. The verification was manual, using Hoare logic, and the algorithms in question were those implemented in the firmware of the T800 Transputer. During the formalization, a few issues in IEEE-754 were found and the verification uncovered bugs that would have been difficult to find with testing [27]. Foreshadowing the issue in the Pentium 1, bugs were found in the Transputer's handling of floating-point, introduced by the translation to machine code and manual "tidying up" [17], suggesting a need to extend the proof chain to the whole development process and a need for greater automation.

The FDIV bug in the Pentium 1 and the cost of the resultant recall spurred the use of machine-checked formal proofs in the design of floating-point hardware [20], [21]. To this end, IEEE-754 was formalized in a variety of interactive theorem provers, including Isabelle [28], HOL [11], HOL Light [19] (used by Intel), ACL2 [25] (used by AMD and Centaur), PVS [24] and Coq [14], [23], [7]. These and related approaches [2] share a number of common characteristics due to the provers they targeted. They are all instances of the axiomatic approach described in Section III; generally reduce floatingpoint numbers to integers and reals; are intended for use in machine checked proofs; and are normally used to verify implementations of floating-point and specific algorithms based on them. In contrast, this work (and its precursor [26]) follows the algebraic approach; builds on computationally simple primitives; and is intended to be a formal reference for automatic theorem provers providing built-in support for reasoning about floating-point arithmetic.

A number of SMT solvers provide support for early versions of our theory by encoding floating-point expressions as bitvector expressions based on the circuits used to implement floating-point operations. To improve performance, they often rely on over and under approximation schemes. To our knowledge, the earliest implementation of this approach was given in the CBMC model checker [9]. The approach is now used in Z3 [16], MathSAT [12], SONOLAR [22] and CVC4 [3], and improving it remains an active area of research [29]. An alternative approach is based on abstract interpretation. It uses intervals or other abstract domains to over-approximate possible models, and a system of branching and learning similar to the SAT algorithm CDCL to narrow these to particular concrete models [18], [8]. There has also been work to integrate the automated prover Gappa [15] into SMT solvers [13], although these solvers are not known to implement the semantics presented in this paper.

\section{REFERENCES}

[1] I. S. Association. IEEE standard for floating-point arithmetic, 2008. http://grouper.ieee.org/groups/754/.

[2] A. Ayad and C. Marché. Multi-prover verification of floating-point programs. In IJCAR'10, pages 127-141. Springer, 2010.

[3] C. Barrett, C. L. Conway, M. Deters, L. Hadarean, D. Jovanović, T. King, A. Reynolds, and C. Tinelli. CVC4. In $C A V^{\prime} 11$, CAV'11, pages 171177. Springer, 2011.

[4] C. Barrett, R. Sebastiani, S. Seshia, and C. Tinelli. Satisfiability modulo theories. In A. Biere, M. J. H. Heule, H. van Maaren, and T. Walsh, editors, Handbook of Satisfiability, volume 185, chapter 26, pages 825885. IOS Press, February 2009.

[5] C. Barrett, A. Stump, and C. Tinelli. The SMT-LIB Standard: Version 2.0. Technical report, Department of Computer Science, The University of Iowa, 2010.

[6] G. Barrett. Formal methods applied to a floating-point number system. IEEE Trans. Softw. Eng., 15(5):611-621, May 1989.

[7] S. Boldo and G. Melquiond. Flocq: A Unified Library for Proving Floating-point Algorithms in Coq. In ARITH'11, pages 243-252. IEEE, July 2011.

[8] M. Brain, V. D'Silva, A. Griggio, L. Haller, and D. Kroening. Interpolation-based verification of floating-point programs with abstract CDCL. In SAS'13, pages 412-432. Springer, June 2013.

[9] A. Brillout, D. Kroening, and T. Wahl. Mixed abstractions for floatingpoint arithmetic. In FMCAD'09. Springer, 2009.

[10] W. S. Brown. A simple but realistic model of floating-point computation. ACM Trans. Math. Softw., 7(4):445-480, Dec. 1981.

[11] V. A. Carreño. Interpretation of IEEE-854 floating-point standard and definition in the HOL system. Technical report, NASA Langley Research Center, 1995.

[12] A. Cimatti, A. Griggio, B. Schaafsma, and R. Sebastiani. The MathSAT5 SMT Solver. In TACAS'13, volume 7795. Springer, 2013.

[13] S. Conchon, G. Melquiond, C. Roux, and M. Iguernelala. Built-in treatment of an axiomatic floating-point theory for SMT solvers. In SMT'12, pages 12-21. EasyChair, 2013.

[14] M. Daumas, L. Rideau, and L. Théry. A generic library for floatingpoint numbers and its application to exact computing. In TPHOL'O1, pages 169-184. Springer, 2001.

[15] F. de Dinechin, C. Lauter, and G. Melquiond. Certifying the floatingpoint implementation of an elementary function using Gappa. IEEE Trans. on Computers, 60(2), 2011.

[16] L. de Moura and N. Bjørner. Z3: An efficient SMT solver. In TACAS'08, pages 337-340. Springer, 2008.

[17] J. Gibbons. Formal methods: Why should I care? - the development of the T800 transputer floating-point unit. In New Zealand Computer Society Conference, pages 207-217, 1993.

[18] L. Haller, A. Griggio, M. Brain, and D. Kroening. Deciding floatingpoint logic with systematic abstraction. In FMCAD'12, pages 131-140, October 2012.

[19] J. Harrison. Floating point verification in HOL light: The exponential function. In $A M A S T^{\prime}$ '97, pages 246-260. Springer, 1997.

[20] J. Harrison. Floating-point verification using theorem proving. In SFM'06, pages 211-242. Springer, 2006.

[21] J. Harrison. Floating-point verification. J. UCS, 13(5):629-638, 2007.

[22] E. V. Jan Peleska and F. Lapschies. Automated test case generation with SMT-solving and abstract interpretation. In NFM'11, LNCS, pages 298312. Springer, April 2011

[23] G. Melquiond. Floating-point arithmetic in the Coq system. Information and Computation, 216:14-23, July 2012.

[24] P. S. Miner. Defining the IEEE-854 floating-point standard in PVS. Technical report, NASA Langley Research Center, 1995.

[25] J. Moore, T. Lynch, and M. Kaufmann. A mechanically checked proof of the AMD5K86TM floating-point division program. IEEE Trans. on Computers, 47(9):913-926, Sep 1998.

[26] P. Ruemmer and T. Wahl. An SMT-LIB theory of binary floating-point arithmetic. In $S M T$ '10, 2010

[27] J. Woodcock, P. G. Larsen, J. Bicarregui, and J. Fitzgerald. Formal methods: Practice and experience. ACM Comput. Surv., 41(4):19:119:36, Oct. 2009.

[28] L. Yu. A formal model of IEEE floating point arithmetic, July 2013. http://afp.sf.net/entries/IEEE_Floating_Point.shtml.

[29] A. Zeljic, C. M. Wintersteiger, and P. Rümmer. Approximations for model construction. In IJCAR'14, pages 344-359. Springer, 2014. 